Leser der MMW können sich mit allen Fragen zur Abrechnung und Praxisführung an Helmut Walbert, Facharzt für Allgemeinmedizin, Würzburg, wenden. Sie erreichen inn jeden Donnerstag von 13 bis 15 Uhr unter der kostenlosen Rufnummer (0800) 2379830 oder per E-Mail: w@lbert.info.

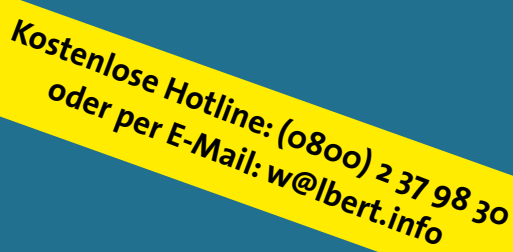

Helmut Walbert

Allgemeinarzt, Medizinjournalist und Betriebswirt Medizin

\title{
Muss ich mir dazu alle notwendigen Geräte anschaffen?
}

\begin{abstract}
Dr. A. M., Allgemeinarzt, Magdeburg: Ich möchte mir möglichst viele Qualifikationsgebundene Zusatzvolumen (OZV) zuordnen lassen. Muss ich die notwendigen Geräte wie Langzeitblutdruckmessgrät, Aufzeichnungsgerät für Langzeit-EKG, Ergometrie-Messplatz selbst vorhalten?
\end{abstract}

Antwort: Insbesondere die Ergometrie erfordert relativ hohe Investitionskosten, die sich wirtschaftlich nicht unbedingt rechnen.

Aus diesem Grund rate ich Ihnen zur Gründung einer Apparategemeinschaft (AG). Hier können sich die Hausarztpraxen in einem bestimmten Einzugsbereich zur Investition im Rahmen einer AG zusammenschließen. Dazu muss nicht unbedingt alles neu erworben werden. Hier können auch vorhandene Geräte in die AG eingebracht werden.

Zur Erbringung der Leistung müssen Sie ausreichend qualifiziert sein. Es muss sichergestellt und festgelegt sein, unter welchen Voraussetzungen Sie im Rahmen der AG über die Geräte verfügen können, um die Leistung ordnungsgemäß zu erbringen. Im Rahmen der KV muss dann mindestens einmal pro Quartal eine entsprechende Leistung erbracht werden, damit Sie ein entsprechendes QZV erhalten. Im Rahmen der Hausarztverträge ist lediglich die Geräteausstattung Voraussetzung, um ein Zusatzbudget zu erhalten.

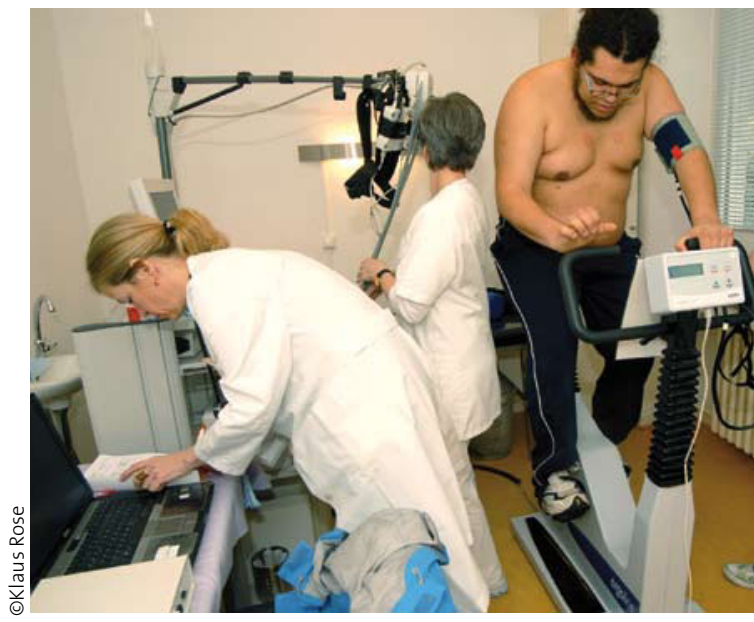

Apparategemeinschaft spart die Anschaffung eines teuren Ergometriemessplatzes.

\section{Arbeitsunfähigkeitsbescheinigung}

\section{Müssen Privatpatienten die selbst bezahlen?}

Dr. med. H. W., Allgemeinarzt, Hamburg: Ich habe gelesen, dass die Arbeitsunfähigkeitsbescheinigung bei privat versicherten Patienten keine Leistung der Krankenversicherung ist. Ist das richtig?

Antwort: Die Ausstellung einer Arbeitsunfähigkeitsbescheinigung (AU) bei privat versicherten Arbeitnehmern ist keine Leistung der Privaten Krankenversicherung. Für die Gehaltsabrechnung bzw. Gehaltsfortzahlung im Krankheitsfall benötigt der
Arbeitgeber die ärztliche Bestätigung. Die allgemeine Regelung „wer bestellt, muss zahlen" gilt auch hier. In einigen Bereichen wird vertraglich festgehalten, dass der Arbeitgeber die Attestkosten übernimmt. Bei der Gesetzlichen Krankenversicherung ist die Vergütung der Ausstellung der AU aufgrund der gesetzlichen Regelungen Teilaufgabe der Krankenkassen. Damit ist die Vergütung über den EBM geregelt. Aber auch hier erhalten freiwillig Versicherte mit Krankentagegeld-Anspruch keine AU nach Muster 1a, sondern ein kostenpflichtiges Attest wie andere „Privatpatienten“. Neben der AU sollte der Arzt gleichzeitig eine „Quittung“ über die Gebühr von $5,36 €(2,3$-fach) ausstellen. Sollte die "glatte" Summe von $5 €$ beim sofortigen Kassieren angenehmer sein, wäre dies der 2,15-fache Satz. Auf jeden Fall sollten solche Kleinbeträge sofort bezahlt werden, da die Ausstellung und Überwachung einer Liquidation in diesen Fällen unverhältnismäßig hohe Verwaltungskosten verursacht. 